Para enlazar con este artículo / To link to this article:

http://dx.doi.org/10.6035/MonTI.2019.11.9

Para citar este artículo / To cite this article:

Ruiz Mezcua, Aurora. (2019) "El triple reto de la interpretación a distancia: Tecnológico, profesional y didáctico." In: Tolosa Igualada, Miguel \& Álvaro Echeverri (eds.) 2019. Porque algo tiene que cambiar. La formación de traductores e intérpretes: Presente \& futuro / Because something should change: Present \& Future Training of Translators and Interpreters. MonTI 11, pp. 243-262.

\title{
EL TRIPLE RETO DE LA INTERPRETACIÓN A DISTANCIA: TECNOLÓGICO, PROFESIONAL Y DIDÁCTICO
}

\author{
Aurora Ruiz Mezcua \\ aurora.ruiz@uco.es \\ Universidad de Córdoba
}

\section{Resumen}

Las nuevas tecnologías permiten ofrecer información a nivel mundial de manera más rápida y eficaz. En este sentido, la interpretación a distancia se ha implantado rápidamente por su efectividad, ya que los intérpretes, sin tener que estar presentes físicamente en un lugar determinado, pueden estar disponibles en cualquier momento utilizando dispositivos tan sencillos y cotidianos, como son un teléfono móvil o un ordenador. De esta forma se ahorra tiempo y dinero para todas las partes implicadas. La interpretación a distancia se está haciendo cada vez más popular a día de hoy, pues permite la comunicación con un intérprete profesional de forma prácticamente instantánea y con resultados satisfactorios. Por ello, se ha empleado ampliamente en contextos profesionales, especialmente dentro de la llamada "interpretación social", que tiene lugar en los hospitales, tribunales, comisarías de policía, servicios de urgencias, centros educativos, etc., aunque quizás ha recibido menos atención en cuestión de formación, al menos hasta la fecha.

\begin{abstract}
"Triple challenge for remote interpreting: technology, profession and teaching"

New technologies have opened up a range of new methods for providing worldwide information which are faster and more effective. In this sense, remote interpretation mode is quickly being promoted for its convenience, as interpreters do not have to be physically present, but can be available at any time using a device as simple and dayto-day as a phone or a computer, saving time and money for all the parties involved. Remote interpretation is becoming increasingly common nowadays, as it enables communication with a professional interpreter almost instantaneously from the distance
\end{abstract}


and with satisfactory results. Remote interpretation has been widely used in many community interpreting settings, such as hospitals, courts, police stations, emergency services, schools, etc., although it has received little attention in terms of training so far.

Palabras clave: Interpretación. Interpretación a distancia. Formación. Interpretación telefónica. Videoconferencia.

Keywords: Interpreting. Remote interpreting. Interpreting training. Telephone interpreting. Videoconference. 


\section{Introducción}

El objetivo principal de este trabajo es analizar la interpretación a distancia desde una perspectiva profesional, didáctica y tecnológica. Las herramientas para interpretar y para formar a intérpretes han cambiado radicalmente esta disciplina desde la aparición de los equipos que se emplean para posibilitar la comunicación oral: empezando por las cabinas de interpretación simultánea y el teléfono para la tele-interpretación, y continuando con Internet, las videoconferencias o las redes sociales, por mencionar algunas.

Antes de la creación de los equipos de interpretación, las técnicas que se empleaban en los diferentes contextos eran la interpretación consecutiva, la susurrada o la bilateral (también conocida como de enlace o dialógica), pero desde el siglo XX se utilizan también la interpretación simultánea y la interpretación remota o a distancia (tanto interpretación telefónica como por videoconferencia), y, en la actualidad, dichos modos están en auge, pues ofrecen numerosas posibilidades para los clientes y una mayor flexibilidad para los contratantes e intérpretes. En este sentido, es importante entender que las tecnologías deben ayudar en el proceso de interpretación y no entorpecerlo y, por tanto, los equipos empleados para la comunicación deben tener la calidad adecuada.

Es frecuente encontrar profesionales que abogan por una mejora de los sistemas, especialmente en lo que a la videoconferencia se refiere. Por ejemplo, en un estudio empírico comparativo realizado en 2010 sobre la interpretación en contexto médico que se efectuó en persona, por teléfono y mediante vídeo, se indica que se requiere más investigación en este último: "additional research on technology also is needed. Bandwidth is still an issue affecting video" (Locatis et al. 2010: 347). Además, la interpretación a distancia ha hecho saltar las alarmas entre profesionales e investigadores en asuntos de especial relevancia, como son la calidad o las condiciones laborales del intérprete:

The actual development of remote and teleconference interpreting has sparked heated debate among practitioners and interpreting scholars and has raised questions of feasibility and working conditions; but the debate has also been linked to the efficiency of service provision and the sustainability of the interpreting profession (Braun 2015: en línea). 
En cualquier caso, la interpretación a distancia, en sus distintos modos, ya se ha implantado a nivel profesional y dispone de estudios científicos y académicos, en cierta medida, como veremos a continuación, encaminados tanto a analizar como a regular esta práctica para que resulte lo más eficaz posible.

[...] la interpretación remota no pretende reemplazar a las modalidades de interpretación in situ: ambos modos pueden coexistir y utilizarse en función de las necesidades concretas de quien recurre a la contratación de servicios de interpretación. En concreto, la interpretación remota ya se solicita hoy día y hay ya un número elevado de empresas que ofrecen este servicio a nivel mundial. De este modo, tanto el sector público como el privado podrán optar por la contratación de servicios de interpretación remota en tanto en cuanto tengan a su disposición determinados medios tecnológicos (Furtado 2014: en línea).

De este modo, en este trabajo, nos proponemos realizar un breve análisis de la interpretación a distancia (interpretación mediada por vídeo e interpretación telefónica) desde el punto de vista tecnológico, profesional y didáctico.

\section{Definición, historia y normativa}

La interpretación a distancia, como su nombre indica, es aquella que se lleva a cabo sin que el intérprete esté presente físicamente durante la conversación multilingüe y, por tanto, se realiza mediante una tecnología que se emplea desde el siglo XX y que abre numerosas posibilidades para el mercado profesional, muchas de ellas aún por explorar.

\subsection{Interpretación mediada por vídeo}

La interpretación a distancia que se lleva a cabo empleando el vídeo se suele denominar "interpretación mediada por vídeo" o "videoconferencia" (en este sentido la vídeo-llamada podría considerarse tanto mediada por vídeo como telefónica, pero suele clasificarse dentro de esta última).

Remote interpreting by videoconference is often simply called remote interpreting when it refers to spoken-language interpreting. In sign-language interpreting, the term video remote interpreting has established itself. Remote interpreting is best described as a method of delivering interpreting. It has been used for simultaneous, consecutive and dialogue interpreting (Braun 2005: en línea).

La primera videoconferencia de la que tenemos constancia, denominada "Symphonie Satellite", se realizó en 1970 en la Organización de Naciones Unidas para conectar las ciudades de París y Nairobi (Esteban Causo 2003: 145). Desde entonces, las organizaciones internacionales siempre han 
mostrado un gran interés por su puesta en marcha. Centros como las Naciones Unidas, la Comisión Europea, el Parlamento Europeo, el Consejo Europeo y la École de Traduction et d'Interprétation (ETI), por ejemplo, han liderado diversos proyectos para su desarrollo (Braun 2015: en línea). De este modo, a día de hoy, la videoconferencia ha mejorado sustancialmente y las asociaciones profesionales de intérpretes abogan por su uso cuando las condiciones técnicas son adecuadas, para realizar interpretación tanto consecutiva como simultánea:

Participants and interpreters must hear and see the same thing, at the same time. Sound-image transmission between the meeting place or places and the interpretation place must be instantaneous and of high quality. [...] Perfect sound-image synchronisation is crucial. [...] For instant and complete understanding of a speaker's message, interpreters need to hear and see the speaker. Therefore, there must be dedicated cameras and operators focusing on the speaker of the moment (AIIC.net 2011: en línea).

Del mismo modo, dentro de los sectores legal y médico, muchos organismos de países europeos emplean la interpretación mediada por videoconferencia y han instalado el equipo necesario en sus salas para que dicha interpretación sea de calidad:

Many European countries have implemented videoconferencing facilities in courtrooms based on the ITU's more recent H.323 standard for videoconferences using the internet, which provides better video and audio quality than ISDN-based systems. Together with high-end peripheral equipment such as cameras and microphones, these systems can provide better support for videoconference-based interpreting than older systems. At the same time, the availability of web- or cloud-based videoconference services providing varying and unstable sound and image quality, and access to them on tablets and other mobile devices (Braun 2015: en línea).

Del mismo modo, se han creado protocolos de actuación estandarizados, instrucciones de uso, normativas (ISO) y glosarios que regulan el empleo de videoconferencias en interpretación para organizadores de eventos e intérpretes.

AIIC's position on the use of videoconferencing, as advised by the Technical and Health Committee, is that an interpreter should not be required to work more than 3 hours a day in a videoconference. If it is scheduled to last longer, manning strength must be increased. Because of the growing relevance of videoconferencing and its impact on conference interpreters, AIIC has drawn up a Code for the Use of New Technologies in Conference Interpretation, available in English which gives more details on how videoconferencing can be used to its best advantage in a multilingual situation (AIIC.net 2000: en línea). 


\subsection{Interpretación telefónica}

Por su parte, la interpretación telefónica es algo más antigua; de hecho, es la forma más antigua de interpretación a distancia, ya que surgió en los años cincuenta del siglo XX (Helguera Gallego et al. 2011: 62). Más concretamente en 1947 dentro del servicio TIS de Australia, estrechamente vinculada a la interpretación social y en respuesta a la gran demanda de comunicación entre la lengua oficial de la nación y otras habladas por población inmigrante (Cabrera 2016: 4). Se conoce como interpretación telefónica a la modalidad de interpretación en la que el servicio se realiza por medio de un teléfono: "Interpretation that is povided via telephone. Also sometimes called overthe-phone interpreting or telephonic interpreting" (Kelly 2008:13). Desde ese momento, otros países también receptores de inmigrantes comenzaron a usar este tipo de interpretación, como decíamos, ligada la interpretación social (en contextos médicos, educativos, tribunales, policiales, servicios de urgencias o violencia de género) y después a crear herramientas específicas para el desarrollo de esta actividad (como es el caso de empresas como CyraCom o Dualia y otras patentes universitarias) y a instaurar protocolos de uso. Así ocurrió, por ejemplo, en Estados Unidos, en California, donde la interpretación telefónica apareció por primera vez, según la investigadora Kelly, en el año 1981:

In the United States, telephone interpreting was first offered in 1981, when a young police officer in San Jose, California, decided to team up with a colleague from the Defense Language Institute in Monterey, California, to form an organization that would help overcome barriers of language .... (Kelly 2008: 5).

Durante la década de los 80 la empresa creada por este policía creció considerablemente y a partir de los 90 empezaron a surgir otras compañías que prestaban servicios de interpretación telefónica y que fueron creciendo con el tiempo, de tal forma que a día de hoy este tipo de interpretación es muy popular en el país.

En España, comenzó a utilizarse entre 2003-2004, momento en el que Dualia introdujo el sistema en algunas instituciones públicas (Ruiz Mezcua 2018: 13). Otras iniciativas y empresas, como Interpret Solutions, se unieron a la implantación de este sistema. Posteriormente se han introducido cambios significativos gracias al uso de los smartphones, por ejemplo, es digna de mención la aplicación Voze, desarrollada por Migralingua (Lázaro Gutiérrez 2012). 


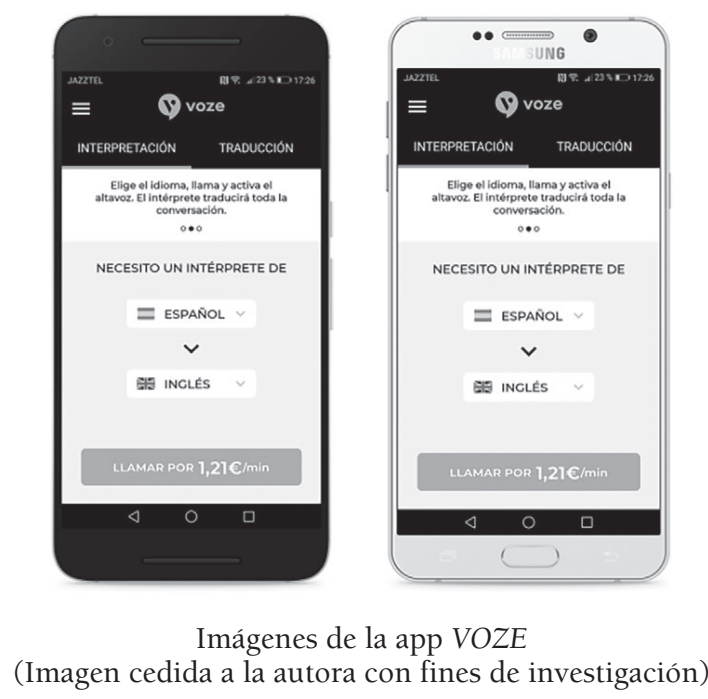

Para el desarrollo del servicio, ha sido imprescindible determinar los perfiles de la población destinataria. Se dirige a los turistas extranjeros en España que hablan uno de los idiomas disponibles en la aplicación [...] También está dirigida a los hispanohablantes que están en un país no hispanohablante [...] Asimismo, la aplicación de interpretación telefónica está pensada para los empresarios que hablan español y que tienen negocios internacionales [...] El equipo [...] estaba compuesto por dos técnicos especializados en el desarrollo de aplicaciones informáticas y de dos traductores profesionales (Empresa Voze 2017: en línea).

\subsection{Ventajas e inconvenientes}

La ventaja más clara que este sistema presenta es la posibilidad de recibir asistencia lingüística desde un punto geográfico hasta otro sin necesitar la presencia física de un intérprete. Esto se vuelve especialmente útil para las empresas, que pueden reducir costes de desplazamiento y obtener el servicio de forma más rápida; los mismos intérpretes, que pueden ofrecer sus prestaciones a clientes de cualquier parte del mundo y ahorrar tiempo (no solo en desplazamientos, sino también por ejemplo, interpretando para varios clientes en un mismo día) y también los usuarios, que obviamente se benefician sobre todo de la inmediatez de la interpretación, especialmente en los casos en los que esta apremia, como ocurre en los servicios de urgencias hospitalarias, asistencia en carreteras o situaciones que no se pueden prever con antelación suficiente. 
Por contrapartida, el intérprete, en ocasiones, y, sobre todo en el caso de la interpretación telefónica, está descontextualizado cuando atiende la llamada y debe servirse fundamentalmente de la información transmitida por la voz (incluidos el tono, dubitativo o no; la presencia o ausencia de balbuceos, la buena dicción o no, la falta o no de coherencia en el mensaje...):

En la interpretación remota, el/la intérprete no puede ver a los/as interlocutores/as ni lo que sucede en el lugar del encuentro (interpretación telefónica) o, incluso disponiendo de imágenes (interpretación por videoconferencias), estas pueden llegarle de manera limitada, abarcando a uno/a solo/a de los/as participantes. Para compensar esta carencia de cuñas visuales, será fundamental servirse de la información transmitida a través de la voz y la prosodia (entonación, tono, pausas, etc.) (SOS-VICs: en línea).

Aunque a priori parece que la interpretación a distancia se está instaurando como solución para algunos contextos en los que se requiere interpretación, esta también cuenta con sus detractores, especialmente cuando empezó a utilizarse con más frecuencia en el mundo profesional. Algunos investigadores y profesionales están en contra del establecimiento de este sistema de interpretación, alegando que el intérprete sufre un mayor estrés durante la interpretación a distancia, al no disponer de una comunicación directa con los hablantes:

Sin embargo, hay que destacar el hecho de que desde que la interpretación remota empezó a proponerse y utilizarse muchos profesionales de la interpretación mostraron una actitud no siempre positiva hacia esta modalidad [...]. Sus reticencias al respecto se deben a diversos motivos [...]: el distanciamiento físico de la ubicación en que transcurre la reunión o conferencia y la falta de comunicación directa con los oradores y los demás intervinientes que conlleva, y que, en opinión de los intérpretes, puede dar lugar a sensaciones de alienación o alejamiento del acto comunicativo (Furtado 2014: en línea).

\subsection{Otras experiencias y estudios}

Conforme a lo indicado con anterioridad y como ya hemos mencionado someramente, en las últimas décadas se están desarrollando ciertos estudios al respecto, avalados por instituciones internacionales de prestigio donde se practica la interpretación, como son la ONU, la UE, la CE, AIIC y diversos centros de enseñanza superior en interpretación. De especial mención son el proyecto conjunto de la International Telecommunication Union (ITU) con la École de Traduction et d'Interpretation (iniciado en abril de 1999) llamado "Assessment of human factors and performance parameters" (Moser-Mercer 2003: en línea); el 3rd Remote Interpretation Test (diciembre de 2005); así como 
el proyecto AVIDICUS 1, 2 y 3 (2008-11) realizado por la Universidad de Surrey y el European Commission's Directorate-General Justice (Braun 2012: en línea). Asimismo, dentro del Programa de Justicia Penal de la UE, también en conjunto con la Universidad de Surrey, surgen los proyectos IVY (Interpreting in Virtual Reality) y EVIVA (Evaluating the Use of Virtual Learning Environments in the Education of Interpreters and their Clients) o el proyecto SHIFT in Orality, desarrollado por esta y otras universidades y empresas europeas y avalado por el programa Erasmus+ de la UE. Todos estos proyectos analizan, entre otras cosas, el desarrollo de la técnica y la viabilidad real de la comunicación mediada por intérpretes a través de medios tecnológicos.

Estos proyectos tenían como objetivo general, precisamente, el estudio y comparación de las modalidades de interpretación in situ e interpretación remota, dado que los intérpretes se quejaban de la existencia de un aumento en la presión psicológica que sufren, así como de un mayor estrés, fatiga, dolores de cabeza, irritabilidad, estrés y otras molestias y dolencias que bien podrían comprometer la calidad de su trabajo, al tiempo que podrían tener serias consecuencias sobre su propia salud en el largo plazo (Furtado 2014: en línea).

Para terminar este apartado, dentro de lo que podríamos denominar también interpretación a distancia, se están llevando a cabo nuevos experimentos profesionales, como la "interpretación telefónica simultánea" propuesta por la empresa londinense Global Lingo. Dicha empresa indica que tan solo se necesita instalar una cabina insonorizada para los intérpretes y una infraestructura de telefonía para que cada participante pueda escuchar el idioma que necesite y hablar sin tener que cambiar de línea.

[...] Afirma Global Lingo que han sabido combinar sus competencias tecnológicas con su experiencia en la interpretación en esta nueva solución: la interpretación telefónica simultánea. El servicio se utilizó por primera vez el mes pasado, el 28 de noviembre, y permitió -según la empresa- una comunicación mucho más fluida (de Rioja, 2017: en línea).

\section{Adaptación de las aulas universitarias a una disciplina cambiante}

Hasta la fecha, las aulas universitarias han instalado laboratorios de idiomas y aulas de cabinas insonorizadas más o menos modernas para enseñar la interpretación consecutiva y la interpretación simultánea, técnicas propias de la interpretación de conferencias. Todos los centros donde se imparte interpretación simultánea en el territorio español disponen de consolas (de diferentes marcas y con mayor o menor cantidad de funciones) para la interpretación 
dentro de sus cabinas, a excepción de las universidades de Alicante y Córdoba, que disponen de un simulador. ${ }^{1}$

Con la irrupción de las nuevas tecnologías en el panorama profesional se espera que los centros de enseñanza superior ofrezcan formación actualizada al respecto y sean caldo de cultivo para fomentar la reflexión y la estandarización de su uso.

Les nouvelles technologies constituent un élément incontournable du paysage professionnel sous la forme de vidéo-conférences et de télé-interprétation (interprétation à distance). Il importe de proposer aux étudiants une expérience directe de ces conditions et d'aménager au sein de la formation un espace de réflexion à leur sujet (Donovan 2010: en línea).

Sin embargo, la interpretación a distancia es, en la mayoría de los casos, una "asignatura pendiente", al menos dentro de los grados de Traducción e Interpretación (donde, por otro lado, se le otorga a la interpretación, como materia y en general, un peso académico muy inferior al de la traducción), pues no existen apenas herramientas docentes creadas específicamente para enseñarla y las que se usan en el mundo profesional o para las enseñanzas de máster no suelen estar disponibles para el alumnado de grado de los centros universitarios. Curiosamente, parece que existen excepciones. Desde los años 50, hay autores que documentan la utilización de teléfonos para la enseñanza específica de la interpretación telefónica ya en 1949 en la Universidad de Mainz, en Germersheim, Alemania (Torres Díaz 2014: 402).

Lo cierto es que hoy día todos estamos familiarizados con las herramientas básicas que se emplean en la interpretación a distancia: ordenadores capaces de admitir la videoconferencia (a través de distintos programas de software libre

1. Las universidades de Alicante y Córdoba son las únicas de España sobre las que tenemos constancia sobre el empleo de un software que simula la consola (Ruiz Mezcua 2010). Esto permite que los alumnos trabajen dentro de las cabinas exclusivamente con ordenadores y no con consolas y pupitres. La universidad de Alicante creó dicho software en 2005 (Ruiz Mezcua 2010: 285) en respuesta al creciente número de alumnos que cursaban interpretación. Se trata de un equipo pionero en su campo y solo instalado en dichos centros, diseñado para convertir cualquier aula de informática en un laboratorio que podía emular las salas de interpretación sin la necesidad de instalar ningún hardware especializado (Ruiz Mezcua 2010: 234 y ss.; Ruiz Mezcua 2012: 320-24). El sistema no está reñido con el uso de consolas reales de interpretación, si bien se entiende que el manejo de estas últimas es completamente necesario en los casos en los que se forme a intérpretes profesionales y la asignatura no sea simplemente una materia obligatoria que todo el alumnado debe cursar. Quizás por ese motivo la Universidad de Alicante ha optado por la desinstalación del equipo y en la actualidad (2018) se emplea un sistema creado por la empresa Televic. En la Universidad de Córdoba aún se sigue utilizando. 
o de pago, como Skype, Adobe, etc.) y telefonía móvil, posiblemente el sistema que más ha evolucionado en los últimos años, convirtiéndose en un dispositivo indispensable que se encuentra en los bolsillos de cualquier adulto y que se emplea cada vez más con fines tanto laborales como lúdicos. Para realizar la interpretación telefónica se emplea un teléfono con manos libres o un terminal al que se le insertan unos receptores o auriculares con control independiente de sonido, de este modo se facilita la conversación a tres.

En una gran cantidad de universidades, sobre todo para enseñanzas superiores (máster, cursos de experto, talleres...) ya se dispone de sistemas para utilizar la videoconferencia y, de esta forma, conectar al profesorado para asistir a reuniones a distancia, dar clases magistrales o participar en tribunales de tesis doctorales. Por ejemplo, en la Facultad de Filosofía y Letras de la Universidad de Córdoba se han defendido con éxito TFG, TFM y tesis doctorales mediante videoconferencia, al menos, desde 2013. Asimismo, en las cabinas de la UML de Londres existen consolas y ordenadores con una cámara instalada para realizar las videoconferencias. Y, más o menos, cada trimestre realizan un debate multilingüe con interpretación sobre un tema, que se escoge de antemano, y este se retransmite por streaming desde su página oficial para todos aquellos que quieran seguirlo.

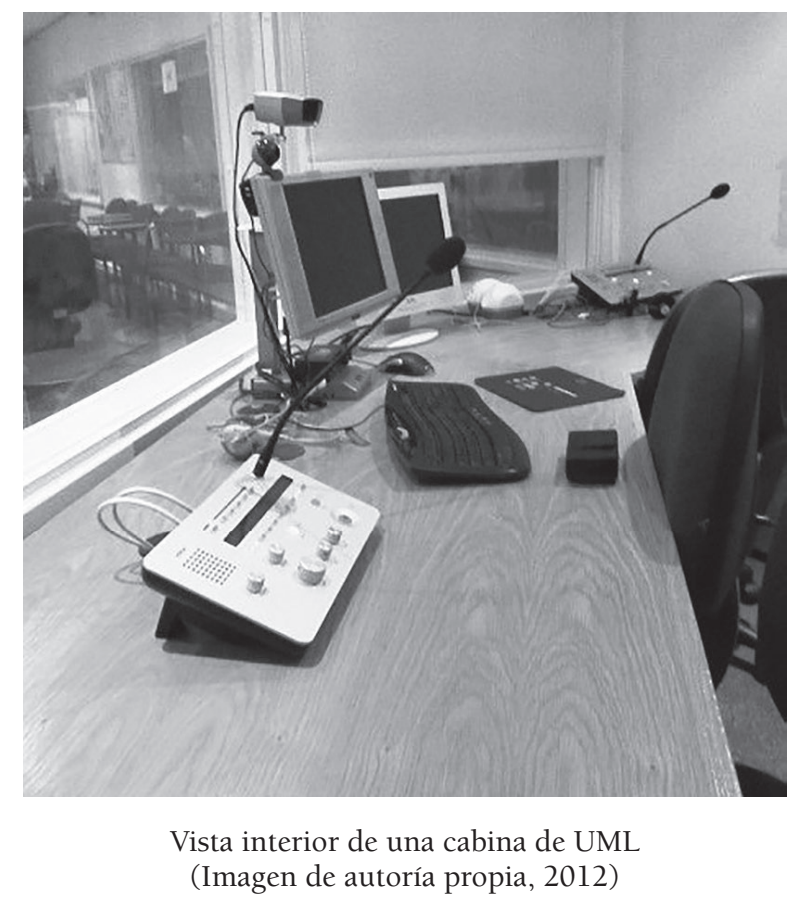


En la Universidad de Ginebra, de las primeras especializadas en la formación de intérpretes, también se cuenta con cabinas equipadas para la enseñanza de la interpretación a distancia: "Our booths are all equipped with computers and screens, speakers are either presenting live or on screen, tablets thus represent additional visual input that is not synchronised with the main input" (Moser-Mercer 2015: en línea), por mencionar alguna de las más prestigiosas. Del mismo modo, hemos podido comprobar que en el Máster en Traducción e Interpretación Especializadas de Oporto se incluye, dentro del marco de la unidad curricular, la denominada "Interpretación Remota y de Teleconferencia" en el Instituto Superior de Contabilidade e Administração de Oporto (ISCAP). Desde que este ciclo de estudios se inició en esta institución, surgió un interés especial por el estudio comparativo tanto de las condiciones de trabajo como del rendimiento de los intérpretes en entornos de interpretación in situ y a distancia, prueba de ello es la tesis doctoral presentada por el doctor Furtado y citada en varias ocasiones en este trabajo (2014: en línea).

El uso de estas herramientas es muy positivo, pero requiere una mayor inversión por parte de los centros, ya que se requiere la presencia de un técnico $y$, en ocasiones, el desplazamiento hasta la zona del campus universitario donde se desarrolla la video-conferencia (que no tiene por qué coincidir con los aularios o las zonas donde se imparten las clases normalmente). Algunos docentes, sobre todo a nivel de grado, se encuentran con la problemática del elevado número de alumnos por clase. Este tipo de actividades requieren un número presencial clausus y, en ocasiones, se sobrepasa el cupo de los que pueden entrar en la sala virtual habilitada al efecto; por eso, prefieren impartir sus clases de forma tradicional y presencial, relegando este sistema a situaciones en las que realmente no puedan desplazarse. No obstante, en prácticamente todas las universidades e instituciones formativas se emplea algún recurso virtual para enseñar la interpretación (aunque sea como herramienta complementaria), como son el Aula Virtual o los Institutos Virtuales, plataformas web (como las plataformas Moodle y similares), programas como Skype...

The Virtualinstitute is a collaborative learning platform that promotes self-regulated learning. The interface allows trainees to have their interpreting performances critiqued by teachers, tutors and peers through uploading their performance files via a web-based dual-track recording functionality (Moser-Mercer 2015: en línea).

Y para la interpretación telefónica se emplean los móviles personales o profesionales o se habilita una línea fija, bien en el aula o bien en la secretaría del centro, para otorgar más realismo a la práctica y simular una situación en la que no tenemos delante al orador y no podemos basarnos en la información visual. 
Por otro lado, también existen otras iniciativas para la modernización de enseñanza de la interpretación, como la creación de repositorios con discursos o software para guiar la interpretación, como los co-financiados por la Comisión Europea, entre los que destacan: Interpretations [P] (1999), creado concretamente en la Universidad británica de Hull, por Annalisa Sandrelli y Jim Hawkis como parte del programa Marie Curie Training and Mobility of Researchers financiado por la Comisión Europea. Este software prototipo fue diseñado para resolver los problemas a los que los alumnos principiantes tenían que enfrentarse en los primeros estadios de su instrucción en la materia (Sandrelli 2005: 5). El primer proyecto que fue llevado a cabo en esta universidad, en 1995, se llamaba "Interpr-It" y su objetivo era trasladar la metodología de la ELAO a la formación de intérpretes en la modalidad de bilateral (Sandrelli 2003: 99). A partir de 2015, este comenzó a utilizarse en combinación con otro software también creado por la misma universidad, llamado Black Box. Este último es similar a los programas que se emplean en los laboratorios de idiomas (por ejemplo, Optimas), ya que consiste en un conjunto de ordenadores conectados a través de la red de área local (LAN) (Sandrelli 2003: 99).

También encontramos ORCIT, el Speechpool (creado por Sophie Llewellyn Smith y Matt Clarke) o NNI, National Network for Interpreting (un proyecto de varias universidades de Reino Unido; la aparición de páginas web diseñadas para la formación específica de la interpretación de conferencias y social, como las desarrolladas por la Universidad de Vigo (Linkinterpreting), la Universidad de Vic (Speak out for Support o SOSVics), la Universidad Pablo de Olavide (Interpretaweb) o la Universidad de Córdoba (que dispone de tres diferentes: Breaking the ICE in Interpretation, INAGR, especializada en el campo de la agroalimentación y, por último, la más antigua, creada en 2012, MAICS). Igualmente, se han desarrollado apps para teléfonos Android, como Cleopatra, la primera aplicación móvil concebida para automatizar los símbolos que se usan en interpretación consecutiva (ConTilde 2018: en línea), aunque no son herramientas diseñadas exclusivamente para la enseñanza de la interpretación a distancia. También es digna de mención otra herramienta virtual llamada Interpreters' Help, creada para gestionar los encargos de interpretación, en este caso quizás pensada sobre todo para profesionales, con el fin de que puedan compartir glosarios, extraer terminología, consultar diccionarios y acceder a una red de trabajo con otros intérpretes.

En el mundo profesional también comienzan a emplearse plataformas de trabajo específicas para la interpretación a distancia, como es Interprefy. Esta última es una herramienta para realizar interpretación por videoconferencia sin 
necesidad de hardware en la que el público puede usar los teléfonos móviles como receptores:

Interprefy provides a premium cloud-based platform for video remote interpreting of conferences, workshops and similar events. Interprefy revolutionizes simultaneous interpreting by using standard IT equipment instead of specialized hardware. Interpreters work remotely from laptops with stable, high-quality sound and video from the event. The audience on site hear their crystal clear voices through smartphones. Participants use their smartphones, tablets and/or laptops, and connect via Wi-Fi or mobile data networks to professional interpreters working remotely. Traditional headsets with standard IR/ radio transmitters can also be used (Interprefy 2018: en línea).

Este tipo de herramientas son las que se englobarían dentro de las llamadas CAIT (Herramientas informáticas para la formación en interpretación):

CAIT (Computer Assisted Interpreter Training) is a relatively new field of Interpreting Studies which began to develop in the mid-1990s. The impetus behind CAIT is an attempt to exploit the multimedia capabilities of Information and Communication Technology (ICT) to enhance the teaching and learning of interpreting in various ways (Sandrelli \& de Manuel 2014: 269).

A pesar de que el empleo de los dispositivos es sencillo y conocido, las técnicas requieren cierto aprendizaje concreto (que hemos mencionado con anterioridad), especialmente, acostumbrarse a trabajar en contextos en los que no solo no se dispone de comunicación no verbal, sino que, por el contrario, debemos percibir los argumentos exclusivamente por la voz y conocer en profundidad los protocolos de actuación que se aplican en las situaciones en las que el intérprete va a trabajar, sabiendo en todo momento cuál es el papel que tiene que desarrollar y dónde está el límite de su actuación. Por lo tanto, se hace patente que los intérpretes deben recibir formación específica que les permita, por ejemplo, manejar el estrés, gestionar los turnos de palabra del resto de usuarios y del suyo propio, tomar o dar la palabra, aprender a hacer preguntas concretas necesarias para la fluidez del diálogo, así como solicitar repeticiones cuando resulte necesario.

\section{A modo de conclusión}

Emily Greene Balch, en uno de sus famosos discursos pronunciado en 1946 con motivo del premio Nobel, ya indicaba que la tecnología nos ofrece sistemas que disminuyen las barreras del tiempo y la distancia, pero al mismo tiempo, la tecnología es solo una herramienta y no una virtud: "Technology gives us the facilities that lessen the barriers of time and distance - the telegraph and cable, the telephone, radio, and the rest. But technology is a tool, not a virtue" 
(Balch 1946: en línea). De este modo, la distancia hoy en día puede ser una ventaja, una forma de acercar mundos y culturas distintas y, en el caso de la interpretación, ha dado pie a la creación de una nueva visión de la profesión, así como a la utilización de herramientas que permiten que los interlocutores no compartan físicamente un espacio, pero sí un sistema de comunicación interlingüe, de forma inmediata, cuando esta resulta imprescindible.

De todos es sabido que la interpretación es una disciplina altamente compleja de realizar y muy vocacional, independientemente del modo o los dispositivos que se empleen. Los medios técnicos han evolucionado enormemente en las últimas décadas, aunque está claro que siempre requieren mejoras y, por ese motivo, siempre han contado con detractores, sobre todo en las primeras fases de su instauración profesional, esto es, en el caso de la interpretación, desde el surgimiento de las cabinas para el modo simultáneo (primero empleado en la OIT y después gracias aquellas cabinas que en Núremberg se describían como "acuarios" y que carecían de insonorización); a los teléfonos duales, equipos portátiles por radio, infrarrojos, aplicaciones, tablets... hasta la libreta de papel más rudimentaria donde se toman notas para recordar las palabras que vamos a traducir de forma oral.

Los centros de enseñanza superior ya se han subido al carro de las tecnologías para poder, humildemente, seguir el ritmo del mercado laboral de los intérpretes, cada vez más interconectados y que, a día de hoy, pueden también realizar su trabajo desde casa (cuestión que antiguamente era impensable) siempre y cuando cuenten con un equipo adecuado. Precisamente, uno de los inconvenientes que más se atribuye a la interpretación a distancia es la dependencia del correcto funcionamiento de los medios tecnológicos. No es una desventaja propia de este modo, pues en el caso de la interpretación simultánea ocurre lo mismo y, sin embargo, se ha probado sobradamente que es una técnica válida que ahorra sobre todo tiempo en la reproducción del discurso meta. Obviamente cualquier servicio profesional siempre debería estar regulado de forma correcta y cumplir con unos estándares de calidad para todos los participantes: clientes, usuarios y trabajadores. Quizás en este sentido la interpretación simultánea va aún un paso por delante.

En cualquier caso, se requieren más estudios e investigaciones para establecer los parámetros óptimos y para subsanar posibles problemas que puedan derivarse del uso de las tecnologías en la interpretación a distancia. Sin lugar a dudas, para que una interpretación sea de calidad, necesitamos contar con los dispositivos adecuados, pero, sobre todo, necesitamos contratar a un intérprete debidamente formado que domine los idiomas, culturas y herramientas que 
deba emplear, que sepa desenvolverse con soltura en el contexto para el que trabaje y, principalmente, que sienta pasión por su profesión.

Las tecnologías, en todos los casos, deben ser aliadas de los profesionales y deben estar a su disposición para facilitar el trabajo, reducir el esfuerzo y promover que estos desempeñen su labor de forma inmejorable. Por eso, a día de hoy la interpretación a distancia supone un reto tecnológico, profesional y didáctico, pero también una realidad que profesionales y docentes debemos explorar.

\section{Bibliografía}

BRAUN, Sabine. (2015) "Remote Interpreting." En: Mikkelson, Holly \& Renée Jourdenais (eds.) 2015. Routledge Handbook of Interpreting. Londres \& Nueva York: Routledge. Versión electrónica: <https://www.researchgate.net/profile/ Sabine_Braun3/publication/280305163_Remote_Interpreting/links/55b0c75f08ae32092e072c71/Remote-Interpreting.pdf>

BRAUN, Sabine \& Judith TAYLOR. (2012) "AVIDICUS comparative studies - part I: Traditional interpreting and remote interpreting in police interviews." En: Braun, Sabina \& Judith. L. Taylor (eds.) 2012. Videoconference and remote interpreting in criminal proceedings. Guildford: University of Surrey, pp. 85-100. Versión electrónica: <http://epubs.surrey.ac.uk/303020/1/05_Braun_Taylor_ comp_study.pdf>

BALCH, G. Emily. (1948). "Toward Human Unity or Beyond Nationalism." En: Varios autores (The Nobel Prize). (1946) Versión electrónica: <https://www. nobelprize.org/prizes/peace/1946/balch/lecture/>

CABRERA, Gabriel. (2016) La interpretación a distancia: nacimiento, maduración y proyección de futuro. Valencia: Universidad Internacional de Valencia. Versión electrónica: <https://www.universidadviu.es/interpretacion-a-distancia/>

DE RiOJA, Lourdes. (2017) "Blog de interpretación." <http://www.bootheando. $\mathrm{com} />$

Donovan, Clare. (2010) "Adapting Training to the Changing Professional Landscape." FORUM. Revue internationale d'interprétation et de traduction/ International Journal of Interpretation and Translation 8:1, pp. 55-71. Versión electrónica: < http://www.jbe-platform.com/content/journals/10.1075/ forum.8.1.03don>

Esteban CAUSO, José A. (2003). "La Interpretación en el siglo 21: desafíos para los profesionales y los profesores de interpretación." En: De Manuel Jerez, Jesús. (coord.) 2003. Nuevas tecnologías y formación de intérpretes. Granada: Atrio, pp.143-185.

FURTADO, Marco Antonio. (2014) A Interpretação In Situ e a Interpretação Remota Realização e Análise Científica de um Conjunto de Estudos Experimentais. Porto: 
Instituto Superior de Contabilidade e Administração do Porto. Tesis doctoral. Versión electrónica: <http://hdl.handle.net/10400.22/4596>

Helguera Gallego, Antonio et al. (2011) "Traducción remota en el servicio de urgencias del hospital Ramón y Cajal del servicio madrileño de salud.” En: Valero Garcés, Carmen (ed.) 2011. Traducción e Interpretación en los Servicios Públicos en un mundo INTERcoNEcTado. Alcalá: Universidad de Alcalá de Henares, pp. 58-66.

KELLY, Nataly. (2008) Telephone Interpreting. A Comprehensive Guide to the Profession. Victoria, BC: Trafford.

LÁZARO GUTIÉRREZ, Raquel. (2012) La interpretación en el ámbito sanitario. Estudio de la asimetría en consultas médicas. Saarbrücken: Editorial Académica Española.

LOCATIS, Craig, et al. (2010) "Comparing in-person, video, and telephonic medical interpretation." Journal of General Internal Medicine 25:4, pp. 345-350.

MARTín RUEL, Estela. (2017) "De la consola a la interpretación automática: una panorámica de la evolución tecnológica en interpretación." En: Ortega Arjonilla, Emilio; Ana Belén Martínez López \& Francisca García Luque (eds.) 2017. Cartografía de la traducción, interpretación y las industrias de la lengua. Granada: Comares.

Moser-Mercer, Barbara. (2003) "Proyecto Remote interpreting: Assessment of human factors and performance parameters." Versión electrónica: <https:// aiic.net/page/1125/remote-interpreting-assessment-of-human-factors-and-pe/ lang/1>

Moser-MerCer, Barbara. (2005) "Remote Interpreting: Issues of Multi-Sensory Integration in a Multilingual Task." Meta 50:2. Versión electrónica: <http:// id.erudit.org/iderudit/011014ar>

Moser-MerCer, Barbara. (2015) "Technology and interpreting: New opportunities raise new questions." OEB Newsportal. Versión electrónica: < https://oeb. global/oeb-insights/interpreting-technology/>

PÖCHHACKER, Franz. (2009) "Issues in Interpreting studies." En: Munday, Jeremy (ed.) 2009. The Routledge companion to translation studies. Londres \& Nueva York: Routledge.

Ruiz MezcuA, Aurora (ed.) (2018) Approaches to telephone interpretation: Research, Innovation, Teaching and Transference. Berna: Peter Lang.

Ruiz MezcuA, Aurora. (2012) "Principales herramientas técnicas para formar intérpretes de simultánea en el panorama universitario español." En: Martí Ferriol, José Luis \& Ana Ma Muñoz Miquel (eds.) 2012. Estudios de Traducción e Interpretación. Entornos de especialidad. Castellón: Universitat Jaume I, pp. 319-327.

RuIz MezCUA, Aurora. (2010) El equipo de interpretación simultánea y sus implicaciones didácticas. Tesis Doctoral. Málaga: Universidad de Málaga (SPICUM). Versión electrónica: <http://riuma.uma.es/xmlui/handle/10630/4732> 
SANDRELli, Annalisa \& Jesús DE MANUEL. (2014) "The Impact of Information and Communication Technology on Interpreter Training." The Interpreter and Translator Trainer 1:2, pp. 269-303. Versión electrónica: <10.1080/1750399X .2007.10798761>

SANDRELLI, Annalisa. (2005) "Designing CAIT (Computer-Assisted Interpreter Training) Tools: Black Box." En: MuTra: Challenges of Multidimensional Translation: Conference Proceedings. Versión electrónica: <http://www.euroconferences.info/proceedings/2005_Proceedings/2005_Sandrelli_Annalisa.pdf>

SANDRELli, Annalisa. (2003) "Herramientas informáticas para la formación de intérpretes: Interpretations y Black Box." En: De Manuel Jerez, Jesús (coord.) 2003. Nuevas Tecnologías y Formación de Intérpretes. Granada: Atrio, pp. 67-112.

VARIOS AUTORES (Shift) (s.d.) "Página de inicio." <https://www.shiftinorality.eu/es> VARIOS AUTORES (Linkinterpreting). (s.d.) "Página de inicio." <http://linkterpreting.uvigo.es/>

VARIOS AUTORES (Interpretaweb). (s.d.) "Página de inicio." <http://www.interpretaweb.es/index.asp>

VARIOS AUTORES (Interpreters' help). (2018) "Página de inicio." < https://interpretershelp.com/how_it_works>

VARIOS AUTORES (National Network for Interpreting). (2018) "Página de inicio." <http://www.nationalnetworkforinterpreting.ac.uk/interactive-resources/>

VARIOS AUTORES (Interprefy). (2018) "Página de inicio." < https://interprefy.com/> VARIOS AUTORES (ORCIT). (2018) "Página de inicio." < https://orcit.eu/>

VARIOS AUTORES (SOS-VIC). (2018) "La interpretación remota en contextos de violencia de género." <http://sosvics.eintegra.es/ la-interpretacion-remota-en-contextos-de-violencia-de-genero>

VARIOS AUTORES (Speechpool). (2018) "Página de inicio." < http://speechpool.net/ es/>

VARIOS AUTORES (ConTilde). (2018) < http://www.traduccionescontilde.com/es/ cleopatra-la-primera-aplicacion-creada-interpretes-consecutiva/>

VARIOS AUTORES (Interpreting in Virtual Reality). (2017) "Página de inicio." < http:// virtual-interpreting.net>

VARIOS AUTORES (INAGR). (2015) "Página de inicio." < http://www.uco.es/inagr/>

VARIOS AUTORES (Breaking the ICE). (2014) < http://www.uco.es/organizacion/ calidad/ice/>

VARIOS AUTORES (MAICS). (2012) "Página de inicio." <http://www.uco.es/maics/ index.php?sec=home>

VARIOS AUTORES (AIIC). (2011) "Using videoconference technology." Versión electrónica: <http://aiic.net/p/5935>

VARIOS AUTORES (AIIC). (2000) "Notes on videoconferencing." Versión electrónica: <http://aiic.net/p/139> 
TORRES DíAZ, Ma . Gracia. (2014). "La interpretación telefónica. El intérprete como coordinador: estudio de unas interacciones telefónicas." Mutatis Mutandis 7:2, pp. 401-417.

VOZE. (2018) Versión electrónica: <https://voze.es/interpretacion-telefonica/>

VOZE. (2018) "Voze App." Versión electrónica: <https://voze.es/app/>

\section{BIONOTA / BIONOTE}

Aurora Ruiz MEzCuA estudió Traducción e Interpretación en la Universidad de Málaga, donde realizó su Tesis Doctoral, titulada "El equipo de la interpretación simultánea y sus implicaciones didácticas" en 2010. Este fue el primer paso para la publicación de sus libros, entre los que destacan Approches to Telephone Interpretation: Research, innovation, Teaching and Tranferece o Apuntes sobre la interpretación simultánea: nacimiento y primeros pasos y artículos como "Interpretación y Formación para los Centros Sanitarios Españoles", "La enseñanza de la interpretación social en el contexto biosanitario: la preparación psicológica del intérprete médico" o "Aplicación práctica de la interpretación judicial a las aulas universitarias". Fue profesora en el York College (UK) durante un año escolar y trabajó como traductora para la empresa Hermes Traducciones y Servicios Linguisticos (un año) y como traductora e intérprete autónoma durante dos años posteriores. Desde el curso 2008/09 trabaja como profesora en la Universidad de Córdoba, donde enseña interpretación en las combinaciones inglés-español, francés-español, así como en varios másteres de la Universidad de Córdoba e ISTRAD (Sevilla). Sus líneas de investigación son la interpretación de conferencias, la interpretación en los servicios públicos y la formación en interpretación.

Aurora RuIz MEZCUA studied Translation and Interpreting at the University of Málaga (Spain), where she completed her Ph.D. in Specialized Translation and Interpreting Studies with a dissertation called "Simultaneous Interpreting Equipment and its Didactical Implications". This was the first step to the publication of most of her books, like Approches to Telephone Interpretation: Research, innovation, Teaching and Tranferece and Apuntes sobre la interpretación simultánea: nacimiento y primeros pasos or her main papers, like "Interpretación y Formación para los Centros Sanitarios Españoles", "La enseñanza de la interpretación social en el contexto biosanitario: la preparación psicológica del intérprete médico" or "Aplicación práctica de la interpretación judicial a las aulas universitarias". She teached Spanish in York College (UK) for a schoolyear. She has also worked as a translator for Hermes Traducciones y Servicios 
Linguisticos and as a freelance translator and interpreter for two years. Since 2008/09 she has been working as a lecturer at Córdoba University (Spain), where she now teaches Simultaneous and Consecutive Interpretation FrenchSpanish, English-Spanish. She also teaches for the Master degree in the same university and ISTRAD (Seville, Spain). Ms. Ruiz research main lines are Conference Interpreting, Community Interpreting and the impact of IT in the teaching and training of interpretation. 